Viscum Oxycedri DC. Ist bei Bakarac (Buccarizza) nicht selten; hier findet man es im Thale Klančina; bei der Höhle Lokvica. Blüht im Monate October und November. Diess Viscum kommt auch bei Hreljin (Piket) vor (Fl. cr. p. 1333!) und hener fand ich es am Calvarienberge bei Buccari und St. Jakob, unweit von Portoré und ist gewiss auch auf anderen Orten, wo Juniperus Oxycedrus wächst, zu finden.

Adenostyles alpina Bluff. et Fingerh. Kommt auch am Fusse des Monte Maggiore, oberhalb von Vela Učka vor. August (1878). Filago germanica L. $\alpha$ ) canescens Coss. Germ. Freyn l. c. p. 357! und $\beta$ ) lutescens Coss. Germ. Beide mit der typischen Form in der Umgebung von Fiume. Juni (1882).

Achillea Millefolium L. var. rosiflora Vuk. Anf sonnigen, buschigen Stellen bei Cavle. Mai, Juni (1882).

Leucanthemum platylepis Borbás in Oesterr. bot. Zeit. XXVIII. pag. 259-261! - Auf steinigen Stellen längs der Strasse, die von Kostrena St. Lucia nach Martinščcica führt. Juni, Juli (1879). Echinops Ritro L. Auch bei Martinšcica. Juli, August (1878).

Carduus candicans W. et K. Im Dragathale bei St. Anna, längs des Weges, der sich von hier nach Orehorica abzweigt, dann auf sonnigen, grasigen Hügeln bei Martinščica. Juni (1880).

Carlina lanata L. Fl. cr. p. 741! - Auf trockenen Wieson und auf Felsen im Dragathale. August bis November (1881).

Centaurea axillaris Willd. $\alpha$ ) integrifolia, $\beta$ ) diversifolia, $\gamma$ ) ochrolepis. Alle drei Varietäten im Dragathale und bei Martinšcica; in schönen und üppigen Exemplaren am westlichen Abhange des Gipfels Solin. Mai bis Juni (1879).

C. amara L. $\beta$. Weldeniana (Rchb. pro species). Freyn l. c. 363! - Auf wüsten Orten bei Martinščica. August, October (1881). C. Scabiosa L. Auch im Dragathale bei St. Anna, nicht häufig. Juni (1880).

(Schluss folgt.)

\title{
Beiträge zur Flora von Kritzendorf in Niederösterreich.
}

Von Moriz Kronfeld.

Polypodium vulgare L. Zahlreich in don Bergwäldern des „Maurer"und "Heuberges".

Lycopodium helveticum L. An dem linksseitigen Rande des Fussweges, der an der Villa "Knaner" vorbei auf den "Langstogerberg" führt.

Typha minima Hoppe. Wio im Vorjahre') traf ich diese für die Flora von Wien als "höchst selten" bezeichnete Pflanze geradezu

1) Cf. „Oest. Botan. Zeitschr." 1881, p. 374. 
gemein auf der grossen, Kritzendorf vorgelagerten Donauinsel, welche im Volksmunde "grosser Ziegelofonhaufen" heisst. Dieser Standort wird sich hoffentlich noch lange für unsere Flora erhalten ${ }^{1}$.

Stencetis bellidiflora A. Br. Auf dem "Langstogerberge".

Erigeron acre L. Sonst in der Wiener Gegend nach Neilreich's Ausdruck "sehr zerstreut und meistens einzeln", aber auf dem "grossen Ziegelofenhaufen " in Menge nebeneinander.

Rudbeckia lacinixta L. Einzeln in dem Theile der Donauau bei Kritzendorf, welcher den Namen "kleiner Rothsam" führt (August 1882).

Artemisia Absinthium L. Auf den Felsen, welche die Burg Greifenstein tragen.

Lappa vulgaris Neilr. In verschiedenen Formen an der Strasse von Kritzendorf nach Höflein. - An trockenen Orten fand ich im Herbste zweimal Exemplare dieser Pflanze mit folgender Abweichung: die Blätter sind gegen das Ende der wenigblüthigen Aeste rosettig zusammengedrängt und zeigen sehr deutlich alle möglichen Stadien der fortschreitenden Metamorphose vom Laubblatte bis zu den widerhakig bewehrten Hüllschuppen. Ich erlaube mir diese Form vorläufig als Lappa vulg. rosulans zu bezeichnen.

Campanula Trachelium L. flore albo. Auf dem „Maurerberg"“ in einem Exemplare neben einem ebenso gearteten von Camp. glomerata L.

Actaea spicata L. Häufig anf dem „Heuberge".

Cakile maritima L. Zu meiner grössten Ueberraschung fand ich ein sehr üppiges Exemplar dieses echt halophyten Gewächses im Donansande des „kleinen Rothsam" schön blühend (Juli 1882). Wahrscheinlich liegt hier eine zufällige (durch Zugvögel bewirkte?) Verschleppung vor.

Parnassia palustris L. In Menge am Wege von Kritzendorf zum "weissen Hof" (namentlich in der Umgebung des von der "Saxonia" einem Corpsstudenten errichteten Grabmals), seltener an dem Wege nach Hadersfeld.

Dianthus superbus L. Oberhalb der Ziegelei auf der bebuschten, sonnigen Anhöhe an dem zum "weissen Hof" führenden Wege.

Myricaria germanica Desv. In dem Weidicht, durch welches der Weg zum Kritzendorfer Bade führt, mit Hippophaë rhamnoides. In grösserer Zahl kommen diese Sträucher auf dem "grossen Ziegelofenhaufen" vor.

Epilobium Dodonei Vill. Es frent mich, für dieso Pflanze wieder einen neuen ${ }^{2}$ ) Standort mittheilen zu können. Auf dem so reichen

1) Ueberhaupt bietet der .grosse Ziegelofenhaufen" auch sonst viel des floristisch Interessanten. Leider beginnt sich aber auf dieser - dem Klosterneuburger Stifte gehörigen -- Insel die Cultur fühlbar zu machen.

") Cf. "Oest. Bot. Zeitschr." 4884, p. 374. 
"Ziegelofenhaufen" kommt auch sie u. zw. ziemlich zahlreich vor. Sonst traf ich sie in der Kritzendorfer Gegend vor dem 1. Steinbruche an dem Wege zum "weissen Hof".

Wien, im November 1882.

\title{
Vorläufige Notiz über Culturversuche mit Orobanchen.
}

\author{
Von B. Stein.
}

Vor etwa zwanzig Jahren wurde in einer gelegentlichen botanischen Plauderei in Breslau der Orobanche apiculata Wallr. gedacht, welche wohl ziem]ich allgemein für eine auf Pelargonien wachsende und durch diese Mutterpflanze gering veränderte Orobanche minor gilt. Freund Haussknecht gab mir damals Samen von $O$. minor, welche or am Rhein auf Trifolium pratense gesammelt hatte, ich säete diese winzigen Samen auf eine der vielen Culturformen des Pelargonium zonale und hatte nach Jahresfrist die Freude, 6-8 kräftige, blühende Exemplare der Orobanche zu sehen. Ob es in der That O. apiculata Wallr. geworden war, weiss ich nicht, und der Versuch hatte weiter keine Folgen, obgleich die Orobanchen jahrelang auf diesem von meiner Mutter mit Pietät gepflegten Pelargoniumtopfe erschienen.

Im Laufe des letzten Winters kam mir der Versuch wieder ins Gedächtniss, und ich beschloss ibn in etwas anderer Richtung neu aufzunehmen. Ich wollte zahlreiche Orobanchen-Arten cultiviren, und indem ich sie nicht jede auf ihre gewohnte Mutterpflanze aussäete, sondern alle Arten auf einer Mutterpflanze, hoffte ich zugleich Resultate zu erlangen, welche über den Werth der zum grossen Theil sehr geringen Art-Differenzen Aufschluss geben mussten. Ich ging von der Ansicht aus, dass die Verschiedenheit der Mutterpflanzen auch geringe Differenzen im Blüthenbau, Farbe etc. der auf ihnen schmarotzenden Arten verursachen könne, wie das bei Orob. minor und $O$. apiculata thatsächlich der Fall zu sein schien. Gelang es mir viele Orobanche-Arten auf einer Nährpflanze zu erziehen, so musste sich zeigen, $a b$ und welche denderungen eintraten, und $o b$ im weiteren Verlaufe nicht etwa ganze Gruppen von Arten besser als Formen einer Art aufuufassen seien.

Von vornherein war die Cultur mehrerer Arten auf einer gemeinsamen einheimischen Nährpflanze aussichtslos, und so entschloss ich mich, die cultivirten Pelargonien (bekanntlich Hybriden aus $\boldsymbol{P}$. inquinans und $P$. zonale) als Mutterpflanze zu wählen. Dieser erste Theil des Versuches ist - vorläufig freilich in bescheidener Weise - gelungen. Mir standen an Samen zu Gebote: Orobanche Hederae Dub., O. speciosa Dietr., O. Ulicis Desml., O. minor Sutt., O. barbata Poir., Phelipaea ramosa (L.), Ph. foliosa Lam. 